\title{
V. Berücksichtigung neuer Beweismittel und Tatsachenvorbringen durch den EGMR
}

\section{Inhaltsverzeichnis}

A. Unterscheidung zwischen echten und unechten Noven als Ausgangspunkt........................ 139

B. Umgang des EGMR mit echten Noven........................................................................ 141

1. Ex-nunc-Prüfung versus ex-tunc-Prüfung in Refoulement-Fällen...................................... 142

a. Zwei typische Refoulement-Konstellationen................................................................ 142

b. Maßgebliche Tatsachengrundlage nach erfolgtem Refoulement.................................. 144

c. Maßgebliche Tatsachengrundlage bei ausstehender Abschiebung.................................. 147

2. Ableitung temporal-beweisrechtlicher Grundsätze.......................................................... 150

C. Umgang des EGMR mit unechten Noven........................................................................... 153

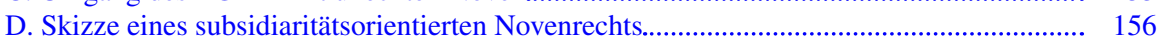

1. Subsidiaritätsrechtliche Kritik an der heutigen Praxis des EGMR.................................... 157

2. Strategien zu einem subsidiaritätsorientierten Umgang mit echten Noven......................... 160

\section{A. Unterscheidung zwischen echten und unechten Noven als Ausgangspunkt}

Der allgemeine verfahrensrechtliche Begriff der Noven geht auf das lateinische Wort novum zurück, das außerhalb des juristischen Sprachgebrauchs allgemein einen neuen Vorfall beziehungsweise im Plural (nova) Neuigkeiten bezeichnet. Hieran angelehnt bezeichnet der juristische Novenbegriff ,,neuen Prozess- und Urteilsstoff“" ${ }^{1}$ Prozessrechtlich gesprochen liegen Noven also vor, wenn während eines hängigen Verfahrens oder aber in Rechtsmittelverfahren neue Rechtsbegehren, neue Tatsachenbehauptungen und Beweismittel oder neue Rechtsstandpunkte vorgebracht werden. Das Novenrecht legt dabei fest, inwiefern solche neue Vorbringen im

\footnotetext{
${ }^{1}$ Vgl. dazu und zum Folgenden Gygi, S. 254.
} 
Rahmen eines einzelnen Verfahrens beziehungsweise im Verlaufe des Instanzenzugs Berücksichtigung finden können; insofern hängt es eng - aber nicht ausschließlich - mit der verfahrensrechtlichen Regelung der Prüfungszuständigkeiten im Instanzenzug zusammen.

In Bezug auf neue Tatsachenbehauptungen wird in der prozessrechtlichen Literatur zwischen echten und unechten Noven unterschieden. Die Unterscheidung bezieht sich auf die Frage, ob die neu vorgebrachten Tatsachen beziehungsweise Beweismittel schon vor einem bestimmten - prozessrechtlich determinierten-Zeitpunkt Bestand hatten oder erst nachträglich entstanden sind. ${ }^{2}$ Während der Begriff der echten Noven Tatsachen und Beweismittel bezeichnet, die erst nach dem maßgeblichen Zeitpunkt entstanden beziehungsweise vorgefunden worden sind, wird der Begriff der unechten Noven für Tatsachen und Beweismittel verwendet, die davor schon Bestand hatten. ${ }^{3}$ Praktisch relevant ist diese Unterscheidung, weil verschiedene Prozessordnungen die Zulässigkeit des neuen Vorbringens maßgeblich von der genannten Differenzierung abhängig machen. ${ }^{4}$

Weder die EMRK noch die EGMR-VerfO knüpfen ihrem Wortlaut nach an die Unterscheidung zwischen echten und unechten Noven an. Im Hinblick auf die teleologische Begründung des Ausschöpfungsgrundsatzes (Art. 35 Ziff. 1 EMRK) drängt sich eine solche Differenzierung jedoch - zumindest für die wissenschaftliche Systematisierung der Rechtsprechung - auf: Die Ausschöpfungsregel verfolgt im Wesentlichen nämlich den Zweck, den Staaten die Möglichkeit zu gewähren, behaupteten Verletzungen der EMRK mit eigenen Mitteln zu begegnen. ${ }^{5}$ Der Ausschöpfungsgrundsatz entbindet die Konventionsstaaten in diesem Sinne davon, sich vor dem EGMR verantworten zu müssen, bevor sie die Möglichkeit erhalten haben, der behaupteten Konventionsverletzung auf innerstaatlicher Ebene zu begegnen. ${ }^{6}$ Dies bedeutet in Bezug auf die Tatsachengrundlagen, dass die Beschwerdeführerin ,den Staat ausdrücklich mit dem als konventionswidrig empfundenen Sachverhalt konfrontieren" muss, ansonsten keine Ausschöpfung des innerstaatlichen Instanzenzugs angenommen werden kann. ${ }^{7}$

Im Hinblick auf diesen Normzweck von Art. 35 Ziff. 1 EMRK ist nun aber von maßgeblichem Unterschied, ob eine Tatsache oder ein Beweismittel schon zum

\footnotetext{
${ }^{2}$ Vgl. Kölz/Häner/Bertschi, Rn. 1019.

${ }^{3}$ Vgl. Kiener/Rütsche/Kuhn, Rn. 1992 f.

${ }^{4}$ Vgl. beispielsweise Art. 229 Abs. 1 der ZPO, wonach in der (erstinstanzlichen) Hauptverhandlung neue Tatsachen und Beweismittel nur noch berücksichtigt werden, wenn sie ohne Verzug vorgebracht werden und erst nach Abschluss des Schriftenwechsels oder nach der letzten Instruktionsverhandlung entstanden oder gefunden worden sind (echte Noven) beziehungsweise bereits vor Abschluss des Schriftenwechsels oder vor der letzten Instruktionsverhandlung vorhanden waren, aber trotz zumutbarer Sorgfalt nicht vorher vorgebracht werden konnten (unechte Noven). Ungenau daher die Begriffsdefinitionen bei Kiener/Rütsche/Kuhn, Rn. 1513, weil suggeriert wird, die Unterscheidung komme nur im Verhältnis verschiedener Instanzen zum Tragen, und außer Acht gelassen wird, dass auch innerhalb des Verfahrens vor einer einzigen Instanz an diese Unterscheidung anknüpfende Bestimmungen zur Zulässigkeit neuer Tatsachenvorbringen zum Tragen kommen können.

${ }^{5}$ Vgl. Lanter, S. 34, m.w.H. auf die Rechtsprechung des EGMR; van der Wilt/Lyngdorf, S. 43.

${ }^{6}$ Vgl. EGMR (Große Kammer), Urteil vom 18. Juli 1999, Selmouni v. Frankreich, § 74.

${ }^{7}$ Vgl. Lanter, S. 77.
} 
Zeitpunkt des letzten innerstaatlichen Entscheids Bestand hatte (unechtes Novum) oder ob sie erst danach entstanden sind (echte Noven). Während es einer Beschwerdeführerin im ersten Fall grundsätzlich möglich war, die zuständigen innerstaatlichen Gerichte mit dem konventionsrechtlich maßgeblichen Sachverhalt zu konfrontieren, ist diese Möglichkeit für den zweiten Fall zu verneinen. Der EGMR tritt auf Beschwerden beziehungsweise Beschwerdeteile, die gestützt auf unechte Noven einen völlig neuen Sachverhalt geltend machen, wohl auch aus dieser Überlegung oftmals gar nicht erst ein; ${ }^{8}$ dies gilt zumindest dann, wenn ein effektives Rechtsmittel zur Verfügung stand, mit dem dieser Sachverhalt den innerstaatlichen Instanzen hätte unterbreitet werden können. ${ }^{9}$ Anders handhabt der EGMR mitunter echte Noven: Unter Berufung auf den Leitgedanken der EMRK, einen effektiven Menschenrechtsschutz zu gewährleisten, berücksichtigt er diese in gewissen Fallkonstellationen, sofern die übrigen Eintretensvoraussetzungen im Zeitpunkt des Beschwerdeeingangs gegeben waren. ${ }^{10}$ Auch wenn der Novenbegriff in der Rechtsprechung des EGMR nicht ausdrücklich auftaucht, drängt es sich vor diesem Hintergrund auf, die Differenzierung zwischen echten und unechten Noven den vorliegenden Betrachtungen zugrunde zu legen.

Angesichts der Tatsache, dass der Novenbegriff vor allem im nationalen Verfahrensrecht und dort oftmals im Zusammenhang der Tatsachenkognition im Instanzenzug verwendet wird, sei den nachfolgenden Ausführungen noch Folgendes vorangestellt: Mit der Verwendung des Novenbegriffs soll nicht suggeriert werden, dass es sich beim EGMR im Verhältnis zu den nationalen Gerichten um eine „,vierte Instanz“ handelt. ${ }^{11}$ Im Vordergrund steht vielmehr die Verwendung praktikabler und verständlicher Begrifflichkeiten - ein Anliegen, dem der Novenbegriff Rechnung zu tragen vermag. Die verwendete Terminologie rechtfertigt sich umso mehr, als der Novenbegriff über verschiedene Rechtsgebiete hinweg Verwendung findet und schon deshalb keine falschen Assoziationen wecken sollte.

\section{B. Umgang des EGMR mit echten Noven}

Nachfolgend ist zunächst aufzuzeigen, wie der EGMR echte Noven handhabt. Untersuchungsgegenstand bildet mit anderen Worten die Frage, wie der EGMR mit Tatsachen und Beweismitteln umgeht, die erst nach dem letzten innerstaatlichen Entscheid

\footnotetext{
${ }^{8}$ Vgl. beispielhaft EGMR, Urteil vom 6. Juli 2017, Sadkov v. Ukraine, § 81, wo der EGMR gewisse Misshandlungsvorwürfe (auch) deshalb als offensichtlich unbegründet zurückwies, weil sie vor den innerstaatlichen Instanzen nicht erhoben worden seien.

${ }^{9}$ Verneint beispielsweise für die unter Art. 3 EMRK vorgebrachte Rüge unmenschlicher Haftbedingungen, zumindest soweit eine Beschwerdeführerin sich noch in Haft befindet; oftmals referenziert in diesem Zusammenhang wird EGMR, Urteil vom 13. November 2014, Papakonstantinou v. Griechenland, § 51 .

${ }^{10} \mathrm{Vgl}$. dazu nachfolgend, V. B.

${ }^{11} \mathrm{Vgl}$. dazu oben, I. A.
} 
entstanden sind. ${ }^{12}$ Subsidiaritätsrechtlich ist diese Fallkonstellation insofern von Interesse, als die innerstaatlichen Instanzen sich mit diesen echten Noven (zumindest im ordentlichen Verfahren, das dem Verfahren vor dem EGMR vorgelagert war) nie auseinandersetzen konnten. Dies hat im konkreten Einzelfall zur Folge, dass die innerstaatlichen Behörden ihrer Primärverpflichtung zum Schutz der Konventionsrechte in Bezug auf diese neuen Sachverhaltselemente gar nicht nachkommen konnten. Es stellt sich deshalb die Frage, ob und gegebenenfalls inwieweit der EGMR in Ausfüllung dieses Vakuums echte Noven wie ein erstinstanzliches Tatsachengericht berücksichtigen soll.

\section{Ex-nunc-Prüfung versus ex-tunc-Prüfung in Refoulement-Fällen}

Die Frage der Berücksichtigung echter Noven betrifft unter Art. 3 EMRK insbesondere Fälle, in denen der EGMR die EMRK-Konformität von Maßnahmen zu prüfen hat, mit welchen eine Person durch ihren Aufenthaltsstaat zum Verlassen des Landes mindestens für eine bestimmte Dauer gezwungen wird. Selbstverständlich ist das Novenrecht neben den Refoulement-Fällen noch in verschiedenen weiteren Fallbereichen von Relevanz. ${ }^{13}$ Nichtsdestotrotz lässt sich am Beispiel der Praxis des EGMR zu Abschiebungsfällen veranschaulichen, welche Kriterien der EGMR anwendet.

\section{a. Zwei typische Refoulement-Konstellationen}

In der Refoulement-Rechtsprechung des EGMR sind im Wesentlichen zwei Fallkonstellationen zu unterscheiden: Wurde die Abschiebung nach dem letzten innerstaatlichen Entscheid vollzogen, kann zum Zeitpunkt des EGMR-Urteils oftmals schon beurteilt werden, ob die innerstaatlich vorgenommene Prognoseentscheidung, dass im Falle einer Rückschiebung kein ernsthaftes Risiko einer unmenschlichen Behandlung im Sinne von Art. 3 EMRK bestehe, sich aus einer ex-post-Sicht faktisch als zutreffend erwiesen hat. Es stellt sich deshalb die Frage, ob der EGMR nachträglich im Zielstaat eingetretene Entwicklungen berücksichtigen darf. Konkret gesprochen: Darf die Tatsache, dass eine Person nach ihrer Abschiebung im Zielstaat eine unmenschliche Behandlung erfahren hat beziehungsweise umgekehrt eine solche gerade ausgeblieben ist, für die Prüfung der konventionsrechtlichen Zulässigkeit der Abschiebung eine Rolle spielen oder nicht? Allgemeiner gesprochen geht es hier darum, ob der EGMR eine innerstaatlich notwendigerweise unter Unsicherheitsbedingungen getroffene Prognoseentscheidung unter Berücksichtigung der darauffolgenden tatsächlichen Geschehnisse auf ihre konventionsrechtliche Rechtmäßigkeit hin überprüfen darf oder ob er diese tatsächlichen Geschehnisse ausblenden muss.

\footnotetext{
${ }^{12} \mathrm{Vgl}$. die eben vorgenommene Abgrenzung zwischen echten und unechten Noven, V. A.

${ }^{13} \mathrm{Vgl}$. dazu nachfolgend, V. B. 2.
} 
Die zweite klassische Konstellation betrifft den Fall, dass eine innerstaatlich letztinstanzlich bestätigte Abschiebung bis zum Urteil des EGMR nicht vollzogen wird, sei es, weil der EGMR nach Eingang einer Beschwerde die Abschiebung gestützt auf Art. 39 EGMR-VerfO bis zum Vorliegen des Urteils durch eine vorsorgliche Maßnahme verboten hat, ${ }^{14}$ sei es, weil der Konventionsstaat aus eigenen Stücken vorläufig darauf verzichtet hat. ${ }^{15}$ Zwischen dem letztinstanzlichen innerstaatlichen Entscheid und dem Urteil des EGMR kann dabei relativ viel Zeit verstreichen: Der EGMR hat im Untersuchungszeitraum 51 Refoulement-Fälle materiell beurteilt; seine durchschnittliche Verfahrensdauer in diesen Fällen betrug rund dreieinhalb Jahre. ${ }^{16}$ In dieser Zeit kann es einer Beschwerdeführerin gelingen, neue Beweise für eine drohende Misshandlung beizubringen. Vor allem aber sind diese dreieinhalb Jahre relativ viel Zeit, wenn man beachtet, wie volatil die Situation sich in verschiedenen Zielstaaten von Abschiebungen (vor allem abgewiesener Asylsuchender) gestaltet. ${ }^{17}$ Auch hier stellt sich also die Frage, ob der EGMR die nach dem letzten innerstaatlichen Entscheid eingetretenen Entwicklungen berücksichtigen darf und soll. Nur schwebt hier im Unterschied zur ersten geschilderten Fallkonstellation weiterhin latent eine Konventionsverletzung im Raume - oder anders gesprochen: die Konventionsverletzung währt (hypothetisch) fort und kann in diesem Sinne nicht als abgeschlossen betrachtet werden. ${ }^{18}$

Nachfolgend werden die beiden Fallkategorien - materielle Prüfung einer Beschwerde durch den EGMR nach erfolgter Abschiebung einerseits und materielle Prüfung einer Beschwerde durch den EGMR vor dem Vollzug der Abschiebung

\footnotetext{
${ }^{14}$ Vgl. für einen solchen Fall statt vieler EGMR, Urteil vom 20. Juni 2017, M.O. v. Schweiz, § 4. Vgl. außerdem EGMR (Große Kammer), Urteil vom 4. Februar 2005, Mamatkulov und Askarov v. Türkei, § 69, wonach die Anordnung einer vorsorglichen Maßnahme insbesondere dann in Betracht kommt, wenn dem EGMR im Zeitpunkt des Beschwerdeeingangs nicht alle Informationen vorliegen, um eine methodisch fundierte Risikoprognose vorzunehmen.

${ }^{15}$ Vgl. für einen solchen (unter Art. 8 EMRK) geprüften Fall EGMR, Urteil vom 16. April 2013, Udeh v. Schweiz, und die diesbezügliche Kritik an der Unterlassung der Schweizer Behörden von Schürer, S. 517.

${ }^{16} \mathrm{Vgl}$. oben, I. D. 3.

${ }^{17}$ In diesem Sinne hält das schweizerische Bundesverwaltungsgericht beispielsweise in seinen Syrien-Urteilen immer wieder fest, ,dass die Situation in Syrien anhaltend instabil und in stetiger Veränderung begriffen“ sei; vgl. statt vieler Urteil des BVGer D-5779/2013 vom 25. Februar 2015 E. 5.2.3 (als Referenzurteil publiziert). Nach Syrien vollzieht die Schweiz derzeit zwar keine Rückschiebungen - die Lage präsentiert sich aber in vielen (oftmals konfliktgeprägten) Entwicklungsländern nicht viel anders. In diesem Sinne hat das schweizerische Bundesverwaltungsgericht für Afghanistan in einem erst kürzlich ergangenen Referenzurteil festgestellt, die Sicherheitslage habe sich landesweit deutlich verschlechtert, vgl. Urteil des BVGer D-5800/2016 vom 13. Oktober 2017 E. 7 (zusammenfassend E. 7.6).

${ }^{18}$ Der EGMR hat bis dato jedoch offengelassen, ob es sich bei der nicht vollzogenen Abschiebung um eine ,fortwährende Situation“ handelt, was implizieren würde, dass die Sechsmonatsfrist für die Einreichung der Beschwerde an den EGMR auch durch einen letztinstanzlichen innerstaatlichen Entscheid nicht zu laufen beginnen würde; vgl. EGMR, Entscheidung vom 17. Oktober 2017, K.L. v. Schweden, $\S \S 24-26$.
} 
anderseits - gesondert dargestellt. Im Vordergrund steht dabei die Frage, ob der EGMR im Sinne eines ex-nunc-Ansatzes in seiner Rechtsprechung auch nach dem letzten innerstaatlichen Entscheid eingetretene Entwicklungen berücksichtigt oder ob er sich auf eine ex-tunc-Prüfung beschränkt und die Rechtmäßigkeit der Abschiebungsverfügung im Zeitpunkt des letzten innerstaatlichen Entscheids beurteilt. ${ }^{19}$

\section{b. Maßgebliche Tatsachengrundlage nach erfolgtem Refoulement}

Die temporal-beweisrechtliche Herangehensweise des EGMR in RefoulementFällen nach erfolgter Abschiebung ist maßgeblich vom 1991 ergangenen Urteil im Fall Cruz Varas und andere v. Schweden geprägt, welchem der folgende Sachverhalt zugrunde lag: Zusammen mit seiner Ehefrau und dem gemeinsamen Sohn hatte der Beschwerdeführer 1987 in Schweden ein Asylgesuch gestellt und dieses im Wesentlichen mit den prekären Lebensbedingungen in seinem Heimatland Chile begründet. Die schwedischen Behörden lehnten die Asylgesuche ab, weil keine asylrechtlich relevante Verfolgung geltend gemacht worden sei; zudem wurde die Wegweisung aus Schweden verfügt. Im Zuge des kurz darauf angestoBenen Vollzugsverfahrens machte der Familienvater gegenüber den schwedischen Behörden mehrmals geltend, ihm drohe bei einer Rückkehr nach Chile politische Verfolgung. Die Gefahr resultiere einerseits daraus, dass er sich in Schweden für die radikale oppositionelle Gruppierung Patriótico Manuel Rodriguez (FPMR) eingesetzt habe. Im Laufe des Verfahrens brachte er als Hauptausreisegrund anderseits vor, schon vor seiner Ausreise aus Chile politisch verfolgt und in diesem Zusammenhang verschiedentlich misshandelt worden zu sein. Die schwedischen Behörden hielten unter Hinweis auf die Unglaubhaftigkeit dieser - ihrer Auffassung zufolge - nachgeschobenen Vorbringen an der Wegweisung fest und schoben den Beschwerdeführer am 6. Oktober 1989 ab. Die Mutter und der Sohn tauchten ab; ihr Verbleib war im Verfahren vor dem EGMR unbekannt. ${ }^{20}$

Mit Blick auf den maßgeblichen Prüfungszeitpunkt hielt der EGMR in den Erwägungen des Cruz Varas-Urteils fest, eine allfällige konventionsrechtliche Verantwortlichkeit des abschiebenden Staates ergebe sich daraus, eine Beschwerdeführerin durch die Abschiebung dem Risiko einer unmenschlichen Behandlung ausgesetzt zu haben. Um das Bestehen dieses Risikos zu beurteilen, ,il faut donc se référer par priorité aux circonstances dont l'État en cause avait ou devait avoir connaissance au moment de l'expulsion, mais cela n'empêche pas la Cour de tenir compte de renseignements ultérieurs; ils peuvent servir à confirmer ou infirmer la

\footnotetext{
${ }^{19} \mathrm{Vgl}$. zu den hier nicht weiter zu vertiefenden praktischen Schwierigkeiten, eine solche ex-tuncSichtweise in Kenntnis späterer Entwicklungen einzunehmen Müller Markus, S. 73 ff. und (konkret zu dieser Schwierigkeit in Refoulement-Fällen) van Drooghenbroeck, S. 267.

${ }^{20}$ Vgl. zum ganzen Sachverhalt EGMR (Plenum), Urteil vom 20. März 1991, Cruz Varas und andere v. Schweden, $\S \S 14-33$.
} 
manière dont la Partie contractante concernée a jugé du bien-fondé des craintes d'un requérant." 21

Gestützt auf diese grundsätzliche ex-tunc-Sicht stellte der EGMR in Bezug auf den konkreten Einzelfall sodann fest, dem Beschwerdeführer sei zwar der Nachweis gelungen, irgendwann in der Vergangenheit Opfer von Misshandlung durch Angehörige des Pinochet-Regimes geworden zu sein. Allerdings hätten die schwedischen Behörden zu Recht Widersprüche in seinen Aussagen zum eigentlichen Ausreisegrund festgestellt. Angesichts des schon vor der Abschiebung angestoßenen demokratischen Wandels in Chile sei ihre Einschätzung der konventionsrechtlichen Zulässigkeit einer Abschiebung deshalb nicht zu beanstanden. ${ }^{22}$ Ergänzend wies er darauf hin, dass es dem Beschwerdeführer auch nach seiner Rückkehr nach Chile nicht gelungen sei, Beweise oder Zeugen für die von ihm geltend gemachten oppositionspolitischen Tätigkeiten zu benennen. ${ }^{23}$

Bis heute ${ }^{24}$ rekurriert der EGMR auf die Grundsätze, die er im Urteil Cruz Varas und andere v. Schweden erstmals abstrakt niedergelegt hat: Im Grundsatz prüft der EGMR ex tunc, ob mit der bereits vollzogenen Abschiebung das ernsthafte Risiko einer unmenschlichen Behandlung einherging. Maßgeblich sind mit anderen Worten im Grundsatz jene Tatsachen, die den verantwortlichen Behörden im Zeitpunkt der Abschiebung bekannt waren, beziehungsweise hätten bekannt sein müssen. Die tatsächlichen Entwicklungen nach der Abschiebung finden nur sekundär Berücksichtigung, wenn sie geeignet sind, die Rechtmäßigkeit der ursprünglichen Einschätzung des Nichtbestehens des Risikos einer unmenschlichen Behandlung durch die innerstaatlichen Behörden zu bestätigen oder zurückzuweisen. Wie eine Minderheit von drei Richtern im Fall Mamatkulov und Askarov v. Türkei zutreffend festgestellt hat, darf den nachträglichen Entwicklungen in diesem Sinne jedenfalls kein entscheidendes Gewicht (,not conclusive ${ }^{6}$ ) zugemessen werden. ${ }^{25}$ In die gleiche Richtung weist die Argumentation des EGMR im Urteil Garabayev v. Russland: Hier stellte er fest, die von der russischen Regierung nicht bestrittenen unmenschlichen Haftbedingungen des Beschwerdeführers in Turkmenistan ,serve to strengthen the Court's above conclusions about a violation of Article 3 by the authorities failure to give a proper consideration to the well-grounded fears raised by the applicant". ${ }^{26}$

\footnotetext{
${ }^{21}$ Vgl. EGMR (Plenum), Urteil vom 20. März 1991, Cruz Varas und andere v. Schweden, $§ 76$ (Hervorhebung durch den Verfasser).

${ }^{22}$ Vgl. EGMR (Plenum), Urteil vom 20. März 1991, Cruz Varas und andere v. Schweden, $\S \S 77-82$.

${ }^{23}$ Vgl. EGMR (Plenum), Urteil vom 20. März 1991, Cruz Varas und andere v. Schweden, § 158.

${ }^{24}$ Vgl. zuletzt EGMR, Urteil vom 26. Januar 2017, X v. Schweiz, § 62 (dort allerdings nicht unter Referenzierung des Urteils im Fall Cruz Varas und andere v. Schweden, sondern von anderen später ergangenen - Urteilen).

${ }^{25}$ Vgl. EGMR (Große Kammer), Urteil vom 4. Februar 2005, Mamatkulov und Askarov v. Türkei, Joint Partly Dissenting Opinion Bratza, Bonello und Hedigan, Ziff. 3.

${ }^{26}$ Vgl. EGMR, Urteil vom 7. Juni 2007, Garabayev v. Russland, no. 38411/02, § 82 (Hervorhebung durch den Verfasser).
} 
Die grundsätzliche ex-tunc-Prüfung ist maßgeblich darauf zurückzuführen, dass sich die völkerrechtliche Verantwortlichkeit des abschiebenden Staates mangels effektiver Kontrolle über die Geschehnisse ${ }^{27}$ im Zielland nur aus der rechtsfehlerhaften Prognoseentscheidung und der gestützt darauf vollzogenen Abschiebung ergeben kann, nicht jedoch aus der durch den Zielstaat zu verantwortenden unmenschlichen Behandlung. ${ }^{28}$ Aus dem Faktum einer nach der Abschiebung erfolgten unmenschlichen Behandlung kann daher nicht auf eine Konventionsverletzung geschlossen werden, ebenso wie ausgeschlossen ist, aus dem Ausbleiben einer unmenschlichen Behandlung nach der Abschiebung unmittelbar auf die Rechtmäßigkeit der Abschiebung zu schließen. Konsequenterweise ist es in der Rechtsprechung des EGMR nach Cruz Varas v. Schweden deshalb vorgekommen, dass keine Verletzung von Art. 3 EMRK festgestellt worden ist, obwohl zumindest nicht ausgeschlossen werden konnte, dass es nach der Abschiebung im Zielstaat zu Misshandlungen gekommen war. ${ }^{29}$ Umgekehrt sind Verletzungen von Art. 3 EMRK mitunter bejaht worden, obwohl nach der Rückschiebung keine Hinweise auf eine unmenschliche Behandlung vorlagen. ${ }^{30}$

In der Umsetzung bedeutet die ex-tunc-Rechtsprechung, dass der EGMR für seinen Entscheid grundsätzlich auf die Tatsachenlage abstellen muss, die den innerstaatlichen Behörden im Zeitpunkt der Abschiebung zugänglich war, beziehungsweise bei richtiger Prüfung zugänglich gewesen wäre. Ähnlich wie Strafrichterinnen, die durch Beweisverwertungsverbote gesperrtes (aber letztlich doch zur Kenntnis genommenes) Beweismaterial nicht nur argumentativ, sondern auch psychologisch unberücksichtigt lassen müssen, ${ }^{31}$ ist der EGMR gehalten, sich in die Lage hineinzuversetzen, in welcher sich die innerstaatlichen Behörden zu einem früheren Zeitpunkt und ohne Kenntnis der späteren Entwicklungen befunden haben.

Die Begründung einiger Urteile legt nahe, dass dies eine durchaus anspruchsvolle Aufgabe ist. Verschiedentlich hat der EGMR nämlich den Entwicklungen nach der Abschiebung - anders als durch die Cruz Varas-Rechtsprechung vorgesehen - zumindest argumentativ großes Gewicht zugemessen: Im Fall Mamatkulov und Askarov v.

\footnotetext{
${ }^{27}$ Vgl. zum ,effective control“-Kriterium Brunner/Fuhrer, S. 93 ff.

${ }^{28}$ Vgl. van Drooghenbroeck, S. 267, m.w.H.

${ }^{29} \mathrm{Vgl}$. EGMR, Urteil vom 30. Oktober 1991, Vilvarajah und andere v. Vereinigtes Königreich, $\S \S 109-116$. Für neuere Entscheide vgl. EGMR, Urteil vom 8. Juli 2014, M.E. v. Dänemark, §§ 62, 64 sowie EGMR, Urteil vom 21. Juli 2015, H.S. und andere v. Zypern, §§ 265-283 und namentlich $\S 282$ : „The Court notes that following the applicants' deportation their representative informed the Court that the majority of the applicants had been arrested and detained upon their return to Syria. Furthermore, one applicant, M.J., claimed that he had been subjected to ill-treatment during their detention. Even assuming, however, that he was subjected to treatment contrary to Article 3 upon his return to Syria at the time of his deportation in 2010, there was no evidence before the domestic authorities or the Court that at the material time the applicant was at risk of being subjected to such treatment [Hervorhebung durch den Verfasser]. “

${ }^{30}$ Vgl. EGMR, Urteil vom 24. Februar 2009, Ben Khemais v. Italien, § 51 und §§ 62-64.

${ }^{31} \mathrm{Vgl}$. zu dieser Schwierigkeit das Interview bei Lorenz.
} 
Türkei hielt der EGMR zu Ungunsten der Beschwerdeführer fest, die türkische Regierung habe medizinische Berichte von usbekischen Gefängnisärzten vorgelegt, aus welchen hervorgehe, dass die Beschwerdeführer nach wie vor unversehrt seien, und verneinte - wesentlich gestützt auf dieses Argument - eine materielle Verletzung von Art. 3 EMRK. ${ }^{32}$ Auch im Fall $Y$ v. Russland erwog der EGMR, es lägen keine Beweise dafür vor, dass der Beschwerdeführer und sein Sohn nach der Abschiebung nach China Opfer einer unmenschlichen Behandlung geworden seien. ${ }^{33}$

Umgekehrt kam der EGMR im Fall Khamidkariyev v. Russland zum Schluss, dass aufgrund der spärlich vorliegenden Informationen davon auszugehen sei, dass die Möglichkeiten des Beschwerdeführers, aus dem usbekischen Gefängnis Kontakt mit der Außenwelt aufzunehmen, stark eingeschränkt seien und dies die Gefahr von Misshandlungen erhöhe. ${ }^{34}$ Deutlich vorsichtiger äußerte sich der EGMR im Fall Ermakov v. Russland, wo er unter Feststellung einer Verletzung von Art. 3 EMRK nur ergänzend darauf hinwies, die Geschehnisse nach der zwangsweisen Ausschaffung des Beschwerdeführers nach Usbekistan würden die Begründetheit der Furcht des Beschwerdeführers vor unmenschlicher Behandlung bestätigen. ${ }^{35}$ Die in Ermakov v. Russland verwendete Formulierung (,,[...] the events following the applicant's forcible return to Uzbekistan can be seen as confirming the wellfoundedness of his fears. [...]." $)^{36}$ ist zu begrüßen, zumal sie auch argumentativ klarstellt, dass in Anknüpfung an die Cruz Varas-Rechtsprechung grundsätzlich eine $e x$-tunc-Sichtweise Platz greift. ${ }^{37}$

\section{c. Maßgebliche Tatsachengrundlage bei ausstehender Abschiebung}

Anders präsentiert sich die Rechtslage, wenn der Abschiebungsvollzug noch aussteht. Diesbezüglicher Leitentscheid ist das Urteil des EGMR im Fall Chahalv. Vereinigtes Königreich, der die noch ausstehende Abschiebung einer Sikh-Familie nach Indien betraf. ${ }^{38}$ Hintergrund des Falls bildete der Konflikt um die Unabhängigkeitsbestrebungen der Sikh in der indischen Provinz Punjab. Der Beschwerdeführer war im Vereinigten Königreich eine der führenden Figuren der Sikh-Gemeinschaft und in diesem Zusammenhang in verschiedene religiöse und politische Aktivitäten involviert. Nichtsdestotrotz wiesen die britischen Behörden

\footnotetext{
${ }^{32}$ Vgl. EGMR (Große Kammer), Urteil vom 4. Februar 2005, Mamatkulov und Askarov v. Türkei, $\S \S 76-77$.

${ }^{33}$ Vgl. EGMR, Urteil vom 4. Dezember 2008, Y v. Russland, § 89. Vgl. dazu De Weck, S. 261 f., m.w.H. auf andere Urteile des EGMR.

${ }^{34}$ Vgl. EGMR, Urteil vom 26. Januar 2017, Khamidkariyev v. Russland, § 143.

${ }^{35}$ Vgl. EGMR, Urteil vom 7. November 2013, Ermakov v. Russland, §§ 206-207.

${ }^{36}$ Vgl. EGMR, Urteil vom 7. November 2013, Ermakov v. Russland, § 206.

${ }^{37}$ Missverständlich die Concurring Opinion von Richter Bianku in EGMR (Große Kammer), Urteil vom 23. März 2016, F.G. v. Schweden, wo die Cruz Varas-Rechtsprechung mit einer ex-nunc-Evaluation in Verbindung gebracht wird.

${ }^{38}$ EGMR (Große Kammer), Urteil vom 15. November 1996, Chahal v. Vereinigtes Königreich.
} 
die Asylgesuche ab, unter anderem mit der Begründung, es sei nicht davon auszugehen, dass seine Aktivitäten in Großbritannien das Interesse der indischen Behörden geweckt hätten. Im Hinblick auf den Vollzug der Wegweisung holten die britischen Behörden zudem eine Stellungnahme der indischen Behörden ein, mit der diese zusicherten, den Beschwerdeführer nicht zu misshandeln und ihm sämtliche Rechte eines indischen Staatsbürgers zu gewähren. ${ }^{39}$

Der EGMR stellte in seinem Urteil zum Chahal-Fall zunächst fest, dass im Unabhängigkeitskonflikt in der Provinz Punjab im Jahr 1992 ein Höhepunkt an Gewalt und Instabilität erreicht worden sei und dann eine gewisse Entspannung eingesetzt habe. Angesichts dieser dynamischen Veränderungen sei der Prüfungszeitpunkt für den Ausgang des Beschwerdeverfahrens von erheblicher Bedeutung. ${ }^{40}$ Der Beschwerdeführer stellte sich auf den Standpunkt, maßgebend sei der Zeitpunkt des letzten innerstaatlichen Entscheids im Juni 1992. ${ }^{41}$ Die britische Regierung hingegen machte geltend, Auslöser einer allfälligen völkerrechtlichen Verantwortlichkeit sei die Abschiebung. Weil der Beschwerdeführer noch nicht abgeschoben worden sei, sei aus Sicht des Zeitpunkts des EGMR-Urteils zu beurteilen, ob mit der Abschiebung das ernsthafte Risiko einer Verletzung von Art. 3 EMRK einhergehe. ${ }^{42}$

Der EGMR gab der britischen Regierung Recht und kam zum Schluss, dass das ernsthafte Risiko einer Verletzung von Art. 3 EMRK durch den EGMR aus Sicht des Zeitpunkts seines Urteils zu prüfen sei, wenn eine Abschiebung noch nicht vollzogen worden sei. Die Sachverhaltsumstände zum Zeitpunkt des letzten innerstaatlichen Entscheids seien nur insofern von Interesse, als sie auch ein Licht auf die Situation im Zeitpunkt des EGMR-Entscheids werfen könnten: ,,[...] although the historical position is of interest in so far as it may shed light on the current situation and its likely evolution, it is the present conditions which are decisive. ${ }^{\text {43 }}$ Im Urteil Salah Sheekh v. Niederlande bezeichnete er diese Herangehensweise ausdrücklich als „ex-nunc-Prüfung“. 44 Der EGMR prüft echte Noven im Sinne dieser ex-nunc-Rechtsprechung nicht nur auf der Sachurteils-, sondern auch auf der vorgelagerten Eintretensebene. In diesem Sinne kommt es auch im Rahmen von Zulässigkeitsentscheidungen vor, dass der EGMR prüft,

\footnotetext{
${ }^{39}$ Vgl. zum ganzen Sachverhalt EGMR (Große Kammer), Urteil vom 15. November 1996, Chahal v. Vereinigtes Königreich, §§ 14-41.

${ }^{40}$ EGMR (Große Kammer), Urteil vom 15. November 1996, Chahal v. Vereinigtes Königreich, $\S 83$.

${ }^{41}$ EGMR (Große Kammer), Urteil vom 15. November 1996, Chahal v. Vereinigtes Königreich, $\S 84$.

${ }^{42}$ EGMR (Große Kammer), Urteil vom 15. November 1996, Chahal v. Vereinigtes Königreich, $\S 85$.

${ }^{43}$ EGMR (Große Kammer), Urteil vom 15. November 1996, Chahal v. Vereinigtes Königreich, $\S 86$.

${ }^{44}$ EGMR (Große Kammer), Urteil vom 11. Januar 2007, Salah Sheekh v. Niederlande, § 136: ,„[...] This further implies that, in assessing an alleged risk of treatment contrary to Article 3 in respect of aliens facing expulsion or extradition, a full and ex nunc assessment is called for as the situation in a country of destination may change in the course of time [Hervorhebung im Original]."
} 
ob die neu entstandenen beziehungsweise zugänglich gewordenen Tatsachen etwas an der Einschätzung der innerstaatlichen Gerichte zu ändern vermögen. ${ }^{45}$

In der Umsetzung kann sich die ex-nunc-Praxis bei weiterhin ausstehender Abschiebung sowohl zu Gunsten als auch zu Ungunsten einer Beschwerdeführerin auswirken. Beispiel für erstere Konstellation ist der Fall Venkadajalasarma v. Niederlande, in dem der EGMR festhielt, angesichts des in Sri Lanka angestoßenen Friedensprozesses sei nicht mehr wahrscheinlich, dass der Beschwerdeführer, der für die vormals separatistische Organisation LTTE nur Aufgaben von untergeordneter Bedeutung ausgeführt hatte, für die sri-lankischen Behörden noch von Interesse sei. $^{46}$ In ähnlicher Art und Weise kam der EGMR im Fall Sow v. Belgien zum Schluss, dass die Beschwerdeführerin zumindest im Zeitpunkt seines eigenen Urteils nicht mehr als junge Frau gelten könne, die im Hinblick auf eine allfällige Beschneidung als besonders schutzbedürftig anzusehen wäre. ${ }^{47}$ Im Fall $J . K . v$. Schweden, in dem die Kammer eine hypothetische Verletzung von Art. 3 EMRK noch verneint hatte, kam die Große Kammer hingegen umgekehrt auch aufgrund der kontinuierlichen Verschlechterung der Situation im Irak zum Schluss, dass dem Beschwerdeführer und seiner Familie aufgrund seiner früheren Tätigkeiten für die amerikanische Armee private Verfolgung drohe und der irakische Staat diesbezüglich nicht schutzfähig sei. ${ }^{48}$

Mitunter ist kritisiert worden, dass der EGMR die ex-nunc-Sicht nicht nur zugunsten, sondern auch zulasten einer Beschwerdeführerin für maßgeblich erklärt. Hauptkritikpunkt ist, dass jede Abschiebungsentscheidung damit konventionsrechtlich zulässig werde, wenn für die Abschiebung nur der richtige Zeitpunkt abgewartet werde. ${ }^{49}$ Impliziert wird mit dieser Kritik jedoch, die Verwaltungsbehörden und -gerichte der Konventionsstaaten würden ,,auf Vorrat“ Abschiebungsverfügungen erlassen, welche die Situation in den Zielländern ganz grundsätzlich nicht berücksichtigen. Dieser Vorwurf ist nicht haltbar: In einem Rechtsstaat ginge es nicht an, dass eine Abschiebung vorsorglich und entgegen der tatsächlichen Gefährdungslage für zulässig erklärt und die Entscheidung über den richtigen Zeitpunkt einfach den Vollzugsbehörden überantwortet würde. Es liegen denn auch keinerlei Anhaltspunkte dafür vor, dass in den Konventionsstaaten eine solche - gegen das Legalitätsprinzip und wohl auch gegen Art. 3 EMRK (prozedural) verstoßende - Praxis vorherrschen würde. Vielmehr sind gerade in jüngster Zeit vermehrt Anstrengungen unternommen worden, rechtskräftige

\footnotetext{
${ }^{45}$ Vgl. zuletzt EGMR, Entscheidung vom 7. November 2017, Xv. Deutschland, § 32, wo explizit festgehalten wird, dass die Informationen, welche nach dem letztinstanzlichen deutschen Urteil (in diesem Fall des Bundesverfassungsgerichts) entstanden beziehungsweise zugänglich geworden seien, durch den EGMR auf ihren Beweiswert hin überprüft werden müssten.

${ }^{46}$ Vgl. EGMR, Urteil vom 17. Februar 2004, Venkadajalasarma v. Niederlande, §§66-68. Kritisch zur Anwendung der ex-nunc-Sichtweise in diesem Fall Delas, S. 266.

${ }^{47}$ Vgl. EGMR, Urteil vom 19. Januar 2016, Sow v. Belgien, § 68.

${ }^{48}$ Vgl. EGMR (Große Kammer), Urteil vom 23. August 2016, J.K. und andere v. Schweden, § 113 sowie $\S \S 120-123$.

${ }^{49}$ Vgl. EGMR, Urteil vom 17. Februar 2004, Venkadajalasarma v. Niederlande, Dissenting Opinion Mularoni.
} 
Abschiebungsverfügungen zeitnah umzusetzen, ${ }^{50}$ was auch deren „Anfälligkeit“ für eine nachträgliche Fehlerhaftigkeit erheblich verkleinert. Soweit eine Abschiebung nicht vollzogen werden kann, liegt dies in der Regel nicht an der Notwendigkeit einer Verzögerung durch die betroffenen Verwaltungsbehörden, sondern an der fehlenden Kooperation der Zielländer beziehungsweise der weggewiesenen Drittstaatsangehörigen. ${ }^{51}$ Insofern besteht kein Anlass, allfällige Veränderungen der Sicherheitslage im betreffenden Zielstaat nur zugunsten der Beschwerdeführerinnen zu berücksichtigen. Vor diesem Hintergrund geht der EGMR zu Recht und in grundsätzlich begrüßenswerter Klarheit davon aus, bei noch nicht vollzogener Abschiebung komme in Bezug auf die Tatsachenfeststellung die ex-nunc-Sicht zum Tragen.

\section{Ableitung temporal-beweisrechtlicher Grundsätze}

In Zusammenfassung des letzten Kapitels kann gesagt werden, dass der EGMR die Rechtmäßigkeit einer bereits vollzogenen Abschiebung im Grundsatz ex tunc aus der Perspektive der innerstaatlichen Behörden im Zeitpunkt der Abschiebung prüft; bei einer noch ausstehenden Abschiebung legt er seiner Prüfung hingegen einen $e x$ nunc-Ansatz zugrunde. ${ }^{52}$ Echten Noven misst er also nur dann entscheidendes Gewicht zu, wenn die Abschiebung weiterhin ausstehend ist; ist die Abschiebung hingegen schon vollzogen worden, können echte Noven allenfalls noch ergänzend herangezogen werden, um das Ergebnis der innerstaatlichen Prognoseentscheidung zu bestätigen oder zurückzuweisen. Im Hinblick auf den hier unternommenen Versuch, allgemeine Grundsätze für den Umgang des EGMR mit echten Noven herauszuarbeiten, fragt sich nun, worin der entscheidende Unterschied zwischen der ersten Konstellation der bereits erfolgten Abschiebung und der zweiten Konstellation der weiterhin ausstehenden Abschiebung besteht.

Einen Ansatzpunkt bildet die Unterscheidung zwischen violations instantanées und „violations continues“, die in der Literatur mitunter verwendet wird..$^{53}$ Mit violation instantanée gemeint sind abgeschlossene, auf ein einzelnes Ereignis (in der Vergangenheit) zurückgehende Konventionsverletzungen. Bezüglich solcher punktueller Konventionsverletzungen wird aus dem Ausschöpfungsgrundsatz (Art. 35 Ziff. 1 EMRK) abgeleitet, dass auf den Sachverhalt im Zeitpunkt des als konventionswidrig gerügten innerstaatlichen Entscheids abzustellen sei. ${ }^{54}$ Davon unterschieden werden violations continues als zeitlich fortgesetzte Konventionsverletzungen; bei der Prüfung solcher Konventionsverletzungen dürfe der EGMR auch jene Tatsachen berücksichtigen, die

\footnotetext{
${ }^{50} \mathrm{Vgl}$. für die Schweiz Botschaft Neustrukturierung Asylbereich, S. 7992 ff. Die Gesetzesrevision bezweckt insbesondere auch eine Beschleunigung des Wegweisungsvollzugs, vgl. dazu Gemperli. ${ }^{51}$ Vgl. dazu im deutschen Kontext Fokusstudie BAMF zum Umgang mit abgelehnten Asylbewerbern in Deutschland, S. $16 \mathrm{ff}$.

${ }^{52} \mathrm{Vgl}$. oben, V. B. 1.

${ }^{53}$ van Drooghenbroeck, S. $265 \mathrm{ff}$.

${ }^{54}$ van Drooghenbroeck, S. 264.
} 
sich erst nach der ursprünglichen Entscheidung ergeben hätten. Falls die Konventionsverletzung auch im Zeitpunkt des Verfahrens vor dem EGMR noch fortwirke, dürften in diesem Sinne auch jene Tatsachen berücksichtigt werden, die sich erst nach dem letzten innerstaatlichen Entscheid ergeben hätten. ${ }^{55}$ In der hier verwendeten Terminologie bedeutet diese dogmatische Differenzierung zwischen violations instantanées und violations continues, dass echte Noven im Verfahren vor dem EGMR insoweit Berücksichtigung finden dürften, als die Konventionsverletzung auch nach dem letzten innerstaatlichen Entscheid noch fortgedauert hat. Eine ex-nunc-Sicht käme hingegen nur dann in Betracht, wenn die Konventionsverletzung auch im Zeitpunkt des Urteils noch fortdauert.

Anders als die eben dargelegte Terminologie suggeriert, liegt das entscheidende Argument für die Berücksichtigung echter Noven jedoch nicht darin, dass es sich bei der geltend gemachten Konventionsverletzung um einen Dauersachverhalt handelt. Entscheidend ist vielmehr, dass die Konventionsverletzung während des Verfahrens vor dem EGMR beziehungsweise im Zeitpunkt des EGMR-Urteils weiterhin latent im Raume steht. Deutlich macht dies gerade das hier vertieft analysierte Beispiel der noch nicht vollzogenen Abschiebungen. Die gegen Art. 3 EMRK verstoßende Abschiebung könnte aufgrund ihrer zeitlichen Konzentration nämlich nur den violations instantanées zugeordnet werden. ${ }^{56}$ Nichtsdestotrotz beurteilt der EGMR die (hypothetische) Rechtmäßigkeit noch nicht vollzogener Abschiebungen ex nunc, weil aufgrund der rechtskräftigen innerstaatlichen Abschiebungsverfügung jederzeit die Realisierung der konventionsrechtlich unter Umständen unzulässigen Abschiebung möglich ist.

Nun bildet die noch nicht vollzogene Abschiebung in der Rechtsprechung des EGMR zwar insofern einen Spezialfall, als sich dieser sonst kaum mit hypothetischen zukünftigen Konventionsverletzungen auseinandersetzt. ${ }^{57}$ Nichtsdestotrotz macht die Fallkonstellation exemplarisch deutlich, dass es nicht das Charakteristikum des Dauersachverhalts ist, das die Berücksichtigung echter Noven rechtfertigt, sondern der Umstand einer weiterhin latent im Raume stehenden Konventionsverletzung. Diese Überlegung lässt sich ohne weiteres auf jene Dauersachverhalte übertragen, die sich auch während des Verfahrens vor dem EGMR noch aktualisieren und insofern konventionsrechtlich latent problematisch bleiben. ${ }^{58}$

$\mathrm{Zu}$ nennen sind in diesem Zusammenhang zunächst Fälle behaupteter unmenschlicher Haftbedingungen. ${ }^{59}$ Dabei handelt es sich angesichts der zeitlich fortgesetzten

\footnotetext{
${ }^{55}$ van Drooghenbroeck, S. 264 f.

${ }^{56}$ Der EGMR seinerseits hat bis dato offengelassen, ob es sich bei der nicht vollzogenen Abschiebung um eine „fortwährende Situation“ handelt. Er hatte die Frage jedoch nicht im Zusammenhang des Ausschöpfungsgrundsatzes (Art. 35 Ziff. 1 EMRK) zu behandeln, sondern im Zusammenhang des Beginns des Fristenlaufs der Sechsmonatsfrist (ebenfalls Art. 35 Ziff. 1 EMRK); vgl. EGMR, Entscheidung vom 17. Oktober 2017, K.L. v. Schweden, §§ 24-26.

${ }^{57}$ Vgl. EGMR (Plenum), Urteil vom 7. Juli 1989, Soering v. Vereinigtes Königreich, § 90.

${ }^{58} \mathrm{Vgl}$. die Darstellung der nachfolgend erwähnten Fälle Lopez Ostra v. Spanien und Rieme v. Schweden bei van Drooghenbroeck, S. $266 \mathrm{f}$.

${ }^{59}$ Diese fallen ebenfalls unter Art. 3 EMRK; vgl dazu oben, I. D. 3.
} 
Haft zweifellos um Dauersachverhalte; die Berücksichtigung echter Noven ist gleichwohl nur denkbar, wenn und soweit die Beschwerdeführerin den beanstandeten Haftbedingungen auch während des Verfahrens vor dem EGMR noch ausgesetzt war. Veranschaulichen lässt sich diese Logik anhand des kürzlich beurteilten Falls Ābele v. Lettland: Der Beschwerdeführer war 2008 wegen eines Tötungsdelikts zu 15,5 Jahren Haft verurteilt worden. Mit einer Beschwerde an den EGMR im Jahr 2012 rügte er aufgrund der Haftbedingungen im Gefängnis „Brasa“ eine Verletzung von Art. 3 EMRK. Der EGMR berücksichtigte in seinem Urteil auch die Geschehnisse nach Erhebung der Beschwerde, ${ }^{60}$ dies jedoch nur bis zum 7. September 2016, als der Beschwerdeführer in ein Gefängnis im lettischen Jēkabpils überführt wurde. ${ }^{61}$

Eine weitere klassische Konstellation fortdauernder und im Moment des EGMRUrteils weiterhin latent vorhandener möglicher Konventionsverletzungen betrifft Immissionen von Industrie- und Verkehrsanlagen. Der Fall López Ostra v. Spanien betraf gesundheitsschädigende Emissionen einer Abfallverbrennungsanlage, die mit staatlichen Zuschüssen und auf öffentlichem Grund direkt in einem Wohngebiet gebaut worden war und zum Zeitpunkt des EGMR-Urteils weiterhin betrieben wurde. ${ }^{62}$ Der EGMR hielt angesichts der fortdauernden Situation ausdrücklich fest, er könne auch Tatsachen (in diesem Fall medizinische Berichte und technische Expertisen) berücksichtigen, die erst nach Einreichung der Beschwerde und nach der Eintretensentscheidung entstanden seien. ${ }^{63}$ Auch im Fall Hatton und andere v. Vereinigtes Königreich, der Lärmimmissionen am Flughafen Heathrow nach dem 1993 revidierten Flugregime zum Gegenstand hatte, stellte der EGMR maßgeblich auf einen 1998 - und damit erst nach Beschwerdeeingang - erschienenen Bericht ab. ${ }^{64}$

Veranschaulichen lässt sich die dargestellte Logik schließlich auch anhand familienrechtlicher Fälle, in denen der EGMR aus dem Blickwinkel von Art. 8 EMRK die Rechtmäßigkeit weiterhin wirksamer innerstaatlicher Entscheidungen zur Kindsunterbringung zu beurteilen hat. Paradigmatisches Beispiel in diesem Zusammenhang bildet der Fall Neulinger und Shuruk v. Schweiz, der die bundesgerichtlich angeordnete Rückführung eines Kindes zu seinem von der Mutter geschiedenen Vater nach Israel zum Gegenstand hatte. ${ }^{65}$ Während eine Kammer des EGMR im Januar 2009 noch zum Ergebnis gekommen war, dass eine Rückführung mit dem Kindswohl zu vereinbaren sei und auch der Mutter eine Rückkehr nach Israel zugemutet werden könne, kam die Große Kammer eineinhalb Jahre später unter ausdrücklichem Hinweis auf die Entwicklungen seit dem Bundesgerichtsurteil im Jahr

\footnotetext{
${ }^{60}$ Vgl. EGMR, Urteil vom 5. Oktober 2017, Ābele v. Lettland, beispielsweise §§ 13, 16, 18-19, 35.

${ }^{61}$ Vgl. EGMR, Urteil vom 5. Oktober 2017, Ābele v. Lettland, § 31.

${ }^{62}$ Vgl. EGMR, Urteil vom 9. Dezember 1994, López Ostra v. Spanien, §§ 7-9.

${ }^{63}$ Vgl. EGMR, Urteil vom 9. Dezember 1994, López Ostra v. Spanien, §§ 44-46.

${ }^{64} \mathrm{Vgl}$. EGMR (Große Kammer), Urteil vom 8. Juli 2003, Hatton und andere v. Vereinigtes Königreich, § 126 (und insbesondere den Verweis auf § 60).

${ }^{65}$ Vgl. EGMR (Große Kammer), Urteil vom 6. Juli 2010, Neulinger und Shuruk v. Schweiz. Gleichgelagerte Fragestellungen haben den EGMR schon in seiner frühesten Rechtsprechung beschäftigt, vgl. EGMR, Urteil vom 22. April 1992, Rieme v. Schweden, namentlich §§ 50-51.
} 
Berücksichtigung echter Noven in Verfahren vor dem EGMR

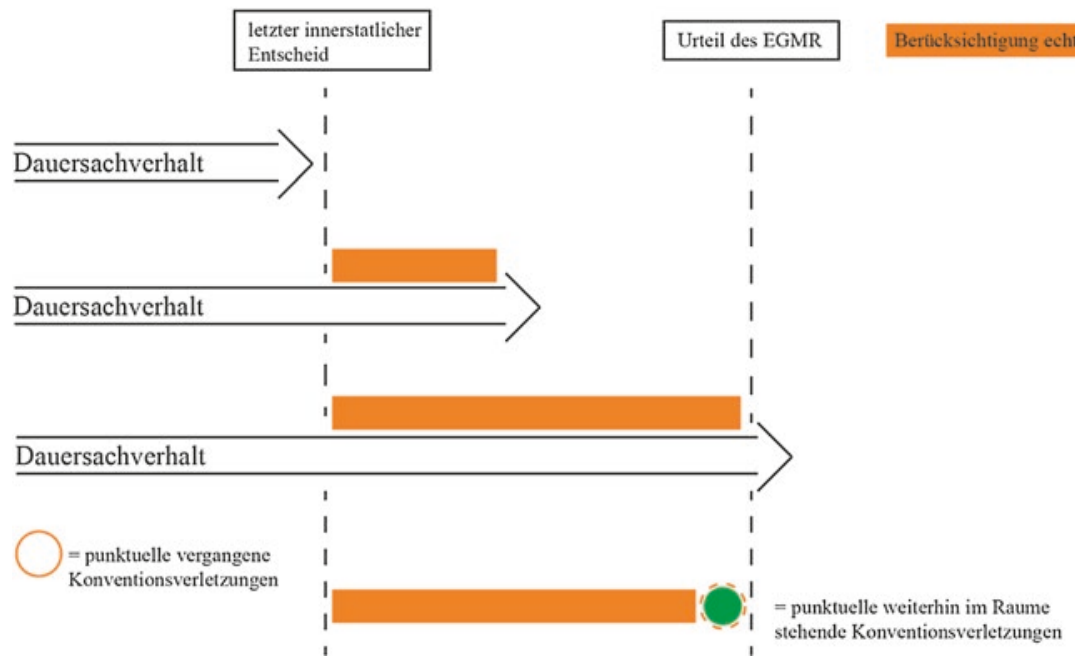

Abb. 1 Schematisierung der Rechtsprechung des EGMR zur Berücksichtigung echter Noven

$2007^{66}$ zum gegenteiligen Schluss und verurteilte die Schweiz wegen einer Verletzung von Art. 8 EMRK. ${ }^{67}$

Zusammenfassend lässt sich also sagen, dass der EGMR echte Noven berücksichtigt, wenn und soweit diese (behauptete) Konventionsverletzungen betreffen, die während des Verfahrens vor dem EGMR weiterhin latent wirksam waren beziehungsweise in naher Zukunft wirksam zu werden drohen. Keine Berücksichtigung finden echte Noven hingegen dann, wenn eine punktuelle in der Vergangenheit liegende Konventionsverletzung im Raume steht beziehungsweise wenn ein Dauersachverhalt bereits während des innerstaatlichen Verfahrens geendet hat. ${ }^{68}$ Bildlich lassen sich diese Grundsätze wie folgt darstellen (Abb. 1):

\section{Umgang des EGMR mit unechten Noven}

Der EGMR hat die Frage, inwieweit er seinen Urteilen unechte Noven zugrunde legen soll, bis anhin - soweit ersichtlich - nie abstrakt erörtert. Dies ist letztlich wohl dem Umstand geschuldet, dass es im Verfahren vor dem EGMR nur selten zu Situationen kommt, in denen unechte Noven im Sinne neu vorgebrachter, aber

\footnotetext{
${ }^{66}$ Vgl. EGMR (Große Kammer), Urteil vom 6. Juli 2010, Neulinger und Shuruk v. Schweiz, § 145. ${ }^{67}$ Vgl. EGMR (Große Kammer), Urteil vom 6. Juli 2010, Neulinger und Shuruk v. Schweiz, § 151. Vgl. zu diesem Fall auch Schürer, S. 516 f.

${ }^{68}$ Dies gilt zumindest dann, wenn das nationale Verfahren den prozeduralen Anforderungen von Art. 3 und Art. 13 EMRK genügt hat.
} 
schon während des innerstaatlichen Verfahrens bestandener Tatsachenbehauptungen materiell überhaupt eine Rolle spielen können:

Nie neu vorgebracht im Sinne der eben in Erinnerung gerufenen Definition sind Tatsachenbehauptungen, die eine Beschwerdeführerin schon den nationalen Instanzen vorgetragen hat. Kommen die nationalen Instanzen aufgrund ihrer Beweiswürdigung zu anderen Tatsachenfeststellungen und hält die Beschwerdeführerin im Verfahren vor dem EGMR an ihrer Schilderung der Geschehnisse fest, liegen in diesem Sinne keine unechten Noven vor. ${ }^{69}$ Von unechten Noven kann aber auch dann nicht die Rede sein, wenn eine Beschwerdeführerin unter Auslassung der innerstaatlichen Instanzen direkt an den EGMR gelangt; in diesem Fall fehlt es an dem begriffsnotwendigen innerstaatlichen Verfahren, in dem die betreffenden Tatsachenbehauptungen hätten vorgebracht werden können. Dies gilt nicht nur für den Fall, dass der EGMR auf eine Beschwerde aufgrund des Ausschöpfungsgrundsatzes (Art. 35 Ziff. 1 EMRK) gar nicht erst eintritt. ${ }^{70}$ Unechte Noven liegen auch dann nicht vor, wenn der EGMR das Vorliegen eines effektiven Rechtsbehelfs verneint und die (erstmals vorgebrachten) Tatsachenbehauptungen einer inhaltlichen Prüfung zuführt. ${ }^{71}$

Unechte Noven liegen hingegen vor, wenn eine Beschwerdeführerin nach Durchlaufen des innerstaatlichen Instanzenzugs an den EGMR gelangt, im innerstaatlichen Verfahren aber nicht sämtliche Tatsachen und Beweismittel vorgebracht hat, die für sie gesprochen hätten. Es liegt auf der Hand, dass diese Konstellation selten ist, weil Beschwerdeführerinnen der innerstaatliche Rechtsschutz eher zugänglich ist als das Verfahren vor dem EGMR und sie daher im Regelfall alles Interesse daran haben, sämtliche für sie sprechenden Argumente ins nationale Verfahren einzubringen. Nichtsdestotrotz ist es vereinzelt vorgekommen, dass Beschwerdeführerinnen die innerstaatlichen Instanzen zwar mit einem konventionsrechtlich relevanten Sachverhalt konfrontiert hatten, diesen dann aber in der Beschwerde an den EGMR um neue, vorbestehende Tatsachenbehauptungen erweiterten. Beispiele hierfür sind die kürzlich entschiedenen Fälle Sadkov v. Ukraine und F.G. v. Schweden.

Im Fall Sadkov v. Ukraine hatte der Beschwerdeführer schon im innerstaatlichen Verfahren vorgebracht, nach einer Verhaftung im Juni 2014 von den diensthabenden

\footnotetext{
${ }^{69}$ In Frage steht hier vielmehr die bereits abgehandelte Frage, wann der EGMR auf Basis des auch den innerstaatlichen Gerichten vorliegenden Beweismaterials von den nationalen Tatsachenfeststellungen abweichen soll; vgl. dazu oben, IV.

${ }^{70} \mathrm{Vgl}$. in diesem Sinne kürzlich EGMR, Entscheidung vom 20. Juli 2017, Atanasov v. Bulgarien und Apostolov v. Bulgarien, mit welcher der EGMR aufgrund von Änderungen der bulgarischen Verfahrensordnung in Abweichung von der früheren Rechtsprechung davon ausging, dass effektive innerstaatliche Rechtsbehelfe zur Verfügung standen, um sich über unmenschliche Haftbedingungen zu beschweren. Davon zu unterscheiden ist EGMR, Urteil vom 5. Oktober 2017, Kormev v. Bulgarien; hier trat der EGMR auf die Beschwerde ein, weil der Schriftenwechsel vor dem EGMR noch durchgeführt worden war, bevor die Gesetzesänderungen in Kraft getreten waren (vgl. § 42).

${ }^{71}$ Vgl. oben, V.A. Das Einschreiten des EGMR im Falle unzureichender innerstaatlicher Tatsachenfeststellung ist subsidiaritätsrechtlich gleichwohl von Interesse; aus diesem Grund auch ist es oben schon abgehandelt worden, vgl. IV. C. 2.
} 
Polizisten geschlagen worden zu sein, um ein Geständnis von ihm zu erwirken. ${ }^{72} \mathrm{Im}$ Verfahren vor dem EGMR brachte er jedoch über diesen Vorwurf hinausgehend vor, die Polizisten hätten ihn sexuell missbraucht, mit Nadelstichen und Elektroschocks malträtiert, an einer Metallstange aufgehängt und ihm mit einer Gasmaske die Luft abgeschnitten. ${ }^{73}$ Diese neuen Tatsachenvorbringen hielt der EGMR aufgrund der Verspätung jedoch für unglaubhaft; er wies das Vorbringen als offensichtlich unbegründet zurück und trat auf die Beschwerde insoweit gar nicht erst ein. ${ }^{74}$

Im Fall F.G. v. Schweden hatte der Beschwerdeführer im ordentlichen Asylverfahren ausdrücklich darauf verzichtet, seine Konversion zum Christentum als möglichen Grund für eine zukünftige Verfolgung im Iran geltend zu machen. ${ }^{75}$ Als er im Rahmen eines außerordentlichen Rechtsmittels auf seinen Verzicht zurückkam, verweigerten die schwedischen Behörden eine inhaltliche Prüfung des Vorbringens, weil es sich nicht um eine „neue Tatsache“ handle. ${ }^{76}$ Bis zuletzt befassten sich die schwedischen Behörden deshalb inhaltlich nicht mit der Frage, ob aus der Konversion für den Fall einer Abschiebung das ernsthafte Risiko einer Verletzung von Art. 3 EMRK hervorgehe. Im Verfahren vor dem EGMR handelte es sich beim Vorbringen der Konversion insofern um ein unechtes Novum, das der Beschwerdeführer zudem mit zahlreichen Beweismitteln unterfütterte, die er den schwedischen Behörden nicht unterbreitet hatte. ${ }^{77}$ Der EGMR enthielt sich vor diesem Hintergrund einer inhaltlichen Prüfung der Beweismittel, hielt aber fest, „qu'il appartient [aux autorités suédoises] de prendre en considération ces éléments, ainsi que toute évolution pouvant intervenir dans la situation générale en Iran et les circonstances propres au cas du requérant". ${ }^{78}$

Sowohl im Fall Sadkov v. Ukraine als auch im Fall F.G. v. Schweden unterzog der EGMR die unechten Noven also keiner eigenständigen inhaltlichen Würdigung. Das Urteilsdispositiv der beiden Urteile unterscheidet sich jedoch maßgeblich: Im Fall Sadkov v. Ukraine trat der EGMR auf die Beschwerde gar nicht erst ein, soweit diese auf unechten Noven basierte. Im Fall F.G. v. Schweden kam er hingegen zum Schluss, dass eine Verletzung von Art. 3 EMRK vorliegen würde, wenn die schwedischen Behörden vor einer allfälligen Abschiebung in den Iran nicht ex nunc die Frage beantworten würden, ob aufgrund der Konversion des Beschwerdeführers das ernsthafte Risiko einer unmenschlichen Behandlung bestehe.

Zwar kann das Urteilsdispositiv im Fall F.G. v. Schweden streng gesehen nicht als Rückweisung an die innerstaatlichen Instanzen verstanden werden, weil sich der EGMR nicht in den nationalen Instanzenzug einordnet. ${ }^{79}$ Inhaltlich entspricht das

\footnotetext{
${ }^{72}$ Vgl. EGMR, Urteil vom 6. Juli 2017, Sadkov v. Ukraine, § 12.

${ }^{73}$ Vgl. EGMR, Urteil vom 6. Juli 2017, Sadkov v. Ukraine, § 10.

${ }^{74}$ Vgl. EGMR, Urteil vom 6. Juli 2017, Sadkov v. Ukraine, § 84. Vgl. für einen vergleichbaren Fall, auf den der EGMR indes in Anwendung des Ausschöpfungsgrundsatzes (Art. 35 Ziff. 1 EMRK) teilweise nicht eintrat EGMR, Urteil vom 20. Oktober 2015, Şakar und andere v. Türkei, §§ 44-46.

${ }^{75}$ Vgl. EGMR (Große Kammer), Urteil vom 23. März 2016, F.G. v. Schweden, §§ 152-153.

${ }^{76}$ Vgl. EGMR (Große Kammer), Urteil vom 23. März 2016, F.G. v. Schweden, §§ 154-155.

${ }^{77}$ Vgl. EGMR (Große Kammer), Urteil vom 23. März 2016, F.G. v. Schweden, § 157.

${ }^{78}$ Vgl. EGMR (Große Kammer), Urteil vom 23. März 2016, F.G. v. Schweden, § 157.

${ }^{79} \mathrm{Vgl}$. oben, I.A.
} 
Vorgehen des EGMR aber einer Rückweisung der Sache an die innerstaatlichen Instanzen zur erneuten Abklärung des rechtserheblichen Sachverhalts. Eine solche „Rückweisung“ dürfte wohl der allgemeinen Herangehensweise entsprechen, wenn eine Beschwerdeführerin dem EGMR unechte Noven vorträgt, die auf eine weiterhin bestehende oder drohende Konventionsverletzung hindeuten. Auch in Bezug auf unechte Noven spielt also die bereits bei den echten Noven vorgenommene Unterscheidung zwischen Konventionsverletzungen, die im Zeitpunkt des EGMR-Urteils abgeschlossen sind, und solchen, die weiterhin latent im Raume stehen. Nur bei letzteren kommt eine „Rückweisung“ in Betracht (Konstellation von F.G. v. Schweden). Beziehen sich die unechten Noven hingegen auf einen Vorgang, der schon während des innerstaatlichen Verfahrens abgeschlossen war, tritt der EGMR in der Regel auf die Beschwerde gar nicht erst ein (Konstellation von Sadkov v. Ukraine). Dies gilt zumindest solange, als es einer Beschwerdeführerin zumutbar gewesen wäre, die unechten Noven schon im innerstaatlichen Verfahren vorzubringen.

\section{Skizze eines subsidiaritätsorientierten Novenrechts}

Der eben skizzierte Umgang des EGMR mit unechten Noven konvergiert mit dem Subsidiaritätsgrundsatz: Bevor eine Beschwerdeführerin an den EGMR gelangt, muss sie die nationalen Instanzen mit dem maßgeblichen Sachverhalt konfrontieren, damit diese eine allfällige Konventionsverletzung schon auf innerstaatlicher Ebene beseitigen können. Selbst wenn weiterhin eine Konventionsverletzung droht, enthält sich der EGMR deshalb einer eigentlichen inhaltlichen Prüfung des unechten Novums und ,übergibt“ die Sache wieder den nationalen Instanzen, die das unechte Novum im Rahmen eines Revisionsverfahrens aber auch tatsächlich prüfen müssen, wollen sie den konventionsrechtlichen Vorgaben nachkommen. Diese Herangehensweise des EGMR ist wohl maßgeblich der Überlegung geschuldet, dass es - im Regelfall - in der Macht der Beschwerdeführerin gestanden hätte, die innerstaatlichen Behörden mit dem als konventionswidrig gerügten Sachverhalt zu befassen. Wo dies nicht der Fall ist, weil die nationale Justiz in der Aufarbeitung der behaupteten Konventionsverletzung versagt hat, steht einer Berücksichtigung unechter Noven nichts im Wege. ${ }^{80}$

Subsidiaritätsrechtlich schwieriger zu beurteilen ist der Umgang des EGMR mit echten Noven. Dass hier neue - womöglich rechtserhebliche - Tatsachen dazugekommen sind, kann weder der Beschwerdeführerin noch dem betreffenden Konventionsstaat angelastet werden, sondern ist dem Zeitablauf geschuldet. Der EGMR befindet sich hier zumindest auf den ersten Blick in einem Spannungsfeld: Gibt er dem effektiven

\footnotetext{
${ }^{80} \mathrm{Vgl}$. beispielsweise EGMR (Große Kammer), Urteil vom 13. Dezember 2010, El-Masri v. Mazedonien, wo der EGMR aufgrund des Versagens der mazedonischen Justiz geradezu gezwungen war, auch Beweise zu berücksichtigen, die der Beschwerdeführer nicht hatte ins nationale Verfahren einbringen können.
} 
Schutz der Konventionsgarantien Vorrang und prüft die Beschwerde auch in Bezug auf die echten Noven? Oder gibt er dem Subsidiaritätsgrundsatz Vorrang und geht in Bezug auf die echten Noven von der Nichtausschöpfung des innerstaatlichen Instanzenzugs aus, weil sich die nationalen Behörden nie mit dem konventionsrechtlich letztlich maßgeblichen Sachverhalt beschäftigen konnten? Wie nachfolgend zu zeigen sein wird, vermag dieses Entweder-Oder-Schema ${ }^{81}$ die komplexe Rechtslage nur unzureichend zu erfassen. Nach einer subsidiaritätsrechtlichen Würdigung des bisherigen Umgangs des EGMR mit echten Noven ist deshalb der Versuch zu unternehmen, alternative Strategien für den Umgang des EGMR mit echten Noven zu entwerfen.

\section{Subsidiaritätsrechtliche Kritik an der heutigen Praxis des EGMR}

Wie oben aufgezeigt worden ist, weist die EMRK die Aufgabe der Tatsachenfeststellung aus subsidiaritätsrechtlichen Überlegungen vorrangig den Institutionen der Konventionsstaaten zu; diese müssen dieser Aufgabe aber auch tatsächlich nachkommen, um dem Verbundzweck Genüge zu tun. Kommen sie der Aufgabe nicht nach, führt dies zwangsläufig zu einem Vakuum, das der EGMR ausfüllen muss, um einen effektiven Menschenrechtsschutz zu gewährleisten. ${ }^{82}$ Werden im Verlaufe eines Verfahrens vor dem EGMR echte Noven entscheidungsrelevant, liegt auf den ersten Blick ein solches Vakuum vor: Die neuen Tatsachen konnten im ausgeschöpften innerstaatlichen Verfahren aufgrund des Zeitablaufs keine Berücksichtigung finden; ein effektiver innerstaatlicher Schutz der Konventionsrechte war insoweit gar nicht möglich. Der EGMR hat daraus zumindest für weiterhin latent im Raume stehende Konventionsverletzungen den Schluss gezogen, seinen eigenen Urteilen eine $e x$-nunc-Sicht zugrunde zu legen. In solchen Fällen rückt der EGMR durch die Berücksichtigung der echten Noven funktional in die Nähe eines „,first-instance [immigration] tribunal“ ${ }^{83}$ Nach hier vertretener Ansicht ist diese ex-nunc-Praxis zwar klar und einfach zu handhaben; subsidiaritätsrechtlich gesehen ist sie jedoch nicht unproblematisch. ${ }^{84}$

\footnotetext{
${ }^{81}$ Mit einem solchen Verständnis offenbar auch Schürer, S. 516.

${ }^{82}$ Allgemein hierzu oben, III. B. 4.

${ }^{83} \mathrm{Vgl}$. die Rede von Jean-Paul Costa, ehemaliger Präsidenten des EGMR, gehalten anlässlich der Eröffnung des Gerichtsjahres 2011 am 28. Januar 2011, in: GB EGMR 2011, S. 35 ff., insbesondere S. 40.

${ }^{84} \mathrm{Vgl}$. in diesem Sinne auch De Weck, S. 269 sowie Delas, S. 272 f. (beide im Hinblick auf die ex-nunc-Praxis des EGMR in Fällen nicht vollzogener Abschiebungen). Die subsidiaritätsrechtliche Problematik wurde auch von Nationalrat Claude Béglé in den parlamentarischen Beratungen zum Bericht des Bundesrates zu den Erfahrungen und Perspektiven des EMRK-Beitritts thematisiert, vgl. Amtl. Bull NR 2016 366: ,,...] la Cour accepte de considérer des faits postérieurs au jugement national, au risque de décrédibiliser les décisions nationales. Un tel procédé risque d'accroître l'insécurité du droit et est donc peu compatible avec le principe de subsidiarité.“
} 
Das Bestehen eines durch den EGMR auszufüllenden Vakuums kann nämlich nicht ohne weiteres angenommen werden. Vielerorts bestehen verfahrensrechtliche Mechanismen, die es erlauben würden, nachträgliche erhebliche Veränderungen der Sachlage (auch während eines am EGMR hängigen Verfahrens) einer innerstaatlichen Prüfung zuzuführen. ${ }^{85}$ Die schweizerische Rechtsprechung beispielsweise hat aus dem verfassungsrechtlichen Verbot der Rechtsverweigerung (Art. $4 \mathrm{aBV}$ ) schon vor Jahrzehnten einen allgemeinen Anspruch auf Wiedererwägung abgeleitet, ,si les circonstances se sont modifiées dans une mesure notable depuis la première décision" ${ }^{86}$ Dieser Anspruch besteht nicht nur, aber insbesondere auch für abgewiesene Asylsuchende, die auf entsprechendes Gesuch hin im Rahmen eines Wiedererwägungsverfahrens (Art. 111b AsylG) neu entstandene Vollzugshindernisse ebenso einer Prüfung zuführen können, ${ }^{87}$ wie im Rahmen eines Zweitasylgesuchs (Art. 111c AsylG) die Prüfung neuer (beziehungsweise neu entstandener) Asylgründe möglich ist. ${ }^{88}$ Mechanismen zur Prüfung neuer Tatsachen bestehen derweil nicht nur in der Schweiz, sondern auch in anderen Konventionsstaaten: Im Fall Nasseri v. Vereinigtes Königreich hat der EGMR eine Beschwerde aus seiner Fallliste gestrichen, weil die britischen Behörden dem Beschwerdeführer in einem zweiten Asylverfahren internationalen Schutz gewährt hatten. ${ }^{89}$

Dabei wäre es zu kurz gegriffen, das Bestehen eines effektiven innerstaatlichen Wiedererwägungsverfahrens nur als Angelegenheit des nationalen Verfahrensrechts zu begreifen. Die in der Schweiz und anderswo bestehenden Wiedererwägungsverfahren bilden vielmehr die Vorgaben ab, die sich für die Konventionsstaaten aus der Konvention selbst ergeben: Art. 1 EMRK und Art. 13 EMRK (und wohl auch die materiellen Konventionsgarantien) verlangen von den Konventionsstaaten nämlich, Verfahrenswege bereitzustellen, die eine Prüfung und allfällige Beseitigung behaupteter Konventionsverletzungen schon auf innerstaatlicher Ebene erlauben..$^{90}$ Diese Vorgabe gilt auch und gerade für Sachverhaltselemente, die erst während oder nach Abschluss eines Verfahrens entstehen und die im Hinblick auf eine weiterhin

\footnotetext{
${ }^{85}$ Vgl. mit Bezug zur Schweiz Aemisegger, S. 74 f.

${ }^{86}$ BGE $100 \mathrm{Ib} 368$ E. 3a. Vgl. zur dogmatischen Herleitung des Anspruchs unter der neuen Bundesverfassung BGE 136 II 177 E. 2.1.

${ }^{87}$ Vgl. noch aus der Rechtsprechung der ARK EMARK 1993/25 E. 3b. Zur Abgrenzung des Wiedererwägungsgesuchs (ausdrücklich normiert in Art. 111b AsylG) vom Folgeasylgesuch (ausdrücklich normiert in Art. 111c AsylG), das ebenfalls die Prüfung von echten Noven erlaubt, die im Hinblick auf Art. 3 EMRK von Relevanz sein können vgl. BVGE 2014/39 E. 4.4 und 4.6.

${ }^{88} \mathrm{Vgl}$. für einen Schweizer Fall, in dem der EGMR nach Eröffnung eines neuerlichen Asylverfahrens eine Beschwerde aus seiner Fallliste gestrichen hat EGMR, Entscheidung vom 18. Oktober 2016, M.G. und M.T. v. Schweiz, §§ 24-31.

${ }^{89}$ Vgl. EGMR, Entscheidung vom 5. November 2015, Nasseri v. Vereinigtes Königreich, §§ 17-20. ${ }^{90} \mathrm{Vgl}$. zur Bedeutung dieser Vorgaben für den maßgeblichen Prüfungszeitpunkt im innerstaatlichen Abschiebungsverfahren Spijkerboer, S. 57 ff.
} 
latent im Raum stehende Konventionsverletzung von Relevanz sein können. ${ }^{91}$ Dabei kann den Behörden aus Praktikabilitätsüberlegungen nicht die Pflicht auferlegt werden, von Amtes wegen tätig zu werden; vielmehr ist es den von einer rechtskräftigen Abschiebungsverfügung betroffenen Personen zuzumuten, ein entsprechendes Gesuch an die zuständigen Behörden zu richten. Allerdings ist für die Effektivität des Rechtsmittels erforderlich, dass aufgrund einer gefestigten Praxis oder entsprechender Gesetzesbestimmungen ein Anspruch auf eine materielle Prüfung der neuen Sachverhaltselemente besteht und dass diese in jenem Verfahren auch tatsächlich berücksichtigt werden. Kommen die Staaten ihrer diesbezüglichen Pflicht nach und besteht innerstaatlich die Möglichkeit, die echten Noven einer effektiven Prüfung zuzuführen, besteht aber subsidiaritätsrechtlich gesprochen kein Vakuum, das der EGMR zur Gewährleistung eines effektiven Menschenrechtsschutzes ausfüllen müsste; das gilt selbst dann, wenn er im konkreten Einzelfall auf die übrigen (nicht auf die echten Noven) gestützten Beschwerdeteile eingetreten ist. ${ }^{92}$ Durch eine Prüfung der echten Noven setzt sich der EGMR vielmehr an die Stelle der nationalen Behörden, ohne dass dafür wirklicher Anlass besteht.

Ein gutes Beispiel für die subsidiaritätsrechtliche Problematik der heutigen Herangehensweise des EGMR bildet der Fall Mohammed v. Österreich. Der Beschwerdeführer hatte 2010 in Österreich ein Asylgesuch gestellt. Das österreichische Bundesasylamt war im Januar 2011 zum Schluss gekommen, dass gemäß der (damals noch anwendbaren) Dublin-II-VO die ungarischen Behörden für die Prüfung des Asylgesuchs zuständig seien, wies das Asylgesuch daher ab und ordnete die Wegweisung des Beschwerdeführers nach Ungarn an. Dieser gelangte ein gutes Jahr später an den EGMR; parallel zum Verfahren vor dem EGMR wurde auf seinen Antrag hin zudem ein zweites Asylverfahren in Österreich eröffnet, das im Wesentlichen die auch medial in der Zwischenzeit in den Fokus gerückten problematischen Verhältnisse des ungarischen Asylsystems zum Gegenstand hatte. ${ }^{93}$ Ohne das Ergebnis dieses zweiten Asylverfahrens abzuwarten, entschied der EGMR, dass eine Abschiebung nach Ungarn materiell keine Verletzung von Art. 3 EMRK zur Folge hätte. ${ }^{94}$ Eine Minderheit von zwei Richterinnen und einem Richter teilte zwar diese Auffassung, hielt in einer Concurring Opinion jedoch fest, dass sie bevorzugt hätten, den

\footnotetext{
${ }^{91}$ Aus diesem Grund auch hat das Bundesgericht festgehalten, es könne nicht von vornherein ausgeschlossen werden, ,dass für die Anwendung von Art. 25 Abs. 3 BV und Art. 3 EMRK rechtserhebliche Sachverhaltselemente im Laufe des Verfahrens eine selbst ungeachtet des bundesgesetzlich verankerten Verbots (echter) Noven (Art. 99 Abs. 1 BGG) zu berücksichtigende Veränderung erfahren können [...], würde doch andernfalls eine im Lichte von Art. 3 EMRK unvollständige Sachverhaltsfeststellung einer Verletzung dieser Bestimmung gleichkommen [...]."Vgl. Urteil des BGer 2C_791/2016 vom 26. September 2016, E. 3.3.

${ }^{92}$ In diesem Sinne wohl auch EGMR, Urteil vom 18. November 2014, M.A. v. Schweiz, Dissenting Opinion Kjølbro, § 6.

${ }^{93} \mathrm{Vgl}$. dazu und zu den weiteren in Österreich eröffneten EGMR, Urteil vom 6. Juni 2013, Mohammed v. Österreich, $\S \S 6-17$.

${ }^{94}$ EGMR, Urteil vom 6. Juni 2013, Mohammed v. Österreich, §§ 91-110.
} 
Ausgang des zweiten österreichischen Asylverfahrens abzuwarten, und es den österreichischen Behörden damit zu ermöglichen, auch aktuelle Entwicklungen im ungarischen Asylsystem und dazu publizierte Berichte zu berücksichtigen. Ausdrücklich nahmen sie in diesem Zusammenhang auch auf das diesbezügliche funktionelle Verhältnis zwischen EGMR und nationalen Gerichten Bezug: „This [das Abwarten der innerstaatlichen Entscheidung] would [...] have ensured greater co-operation between the Court and the national authorities in terms of shared responsibility as the latter are better placed to analyse and decide such matters in the first place. ${ }^{695}$

$\mathrm{Zu}$ den subsidiaritätsrechtlichen Überlegungen kommen handfeste Praktikabilitätsprobleme: Die Aufgabe der Tatsachenfeststellung ist allgemein äußerst zeit- und personalintensiv. Wie der ehemalige Präsident des EGMR, Jean-Paul Costa, in einer 2011 gehaltenen Rede ausgeführt hat, beansprucht der ex-nunc-Ansatz des EGMR in Refoulement-Fällen angesichts der ohnehin bestehenden Ressourcenknappheit am EGMR ,,[...] an excessive portion of its time and human resources, to the detriment of the examination of cases on the merits". ${ }^{66}$ Der Aufwand ist aus subsidiaritätsrechtlicher Sicht zwar nicht zu beanstanden, wenn und soweit nur durch den ex-nunc-Ansatz ein effektiver Schutz der Konventionsrechte sichergestellt werden kann. Nicht nachzuvollziehen ist die Inkaufnahme der Überbelastung durch Anwendung einer $e x$-nunc-Optik jedoch, wenn auf nationaler Ebene Verfahren bereitstehen würden oder sogar angehoben worden sind, die eine Berücksichtigung der echten Noven im Hinblick auf einen effektiven Schutz von Art. 3 EMRK erlauben.

\section{Strategien zu einem subsidiaritätsorientierten Umgang mit echten Noven}

Einen Denkanstoß für einen alternativen Umgang des EGMR mit echten Noven bietet das von Richter Bianku im Fall J.K. und andere v. Schweden in einer Concurring Opinion entwickelte Zwei-Stufen-Vorgehen. ${ }^{97}$ Bianku plädierte dort dafür, dass der EGMR die maßgeblichen Tatsachengrundlagen in Abschiebungsfällen nur dann erneut einer vollumfänglichen Prüfung unterziehe, ,when the national authorities have not conducted a Convention-compliant assessment of the concrete circumstances of the case $[\ldots .]^{\prime .}{ }^{98}$ Bevor der EGMR eine de-novo-Prüfung durchführt, muss er nach dieser Logik zum Schluss kommen, dass die nationalen Behörden den prozeduralen Garantien der EMRK bei der Feststellung des

\footnotetext{
${ }^{95}$ Vgl. EGMR, Urteil vom 6. Juni 2013, Mohammed v. Österreich, Concurring Opinion Berro-Lefèvre, Laffranque und Møse, Ziff. 7.

${ }^{96} \mathrm{Vgl}$. die Rede von Jean-Paul Costa, ehemaliger Präsident des EGMR, gehalten anlässlich der Eröffnung des Gerichtsjahres 2011 am 28. Januar 2011, in: GB EGMR 2011, S. 35 ff., insbesondere S. 40.

${ }^{97} \mathrm{Vgl}$. dazu schon oben, IV. C. 2.

${ }^{98}$ Vgl. EGMR (Große Kammer), Urteil vom 23. August 2016, J.K. v. Schweden, Concurring Opinion Bianku.
} 
Sachverhalts nicht vollumfänglich Rechnung getragen haben. Zwar wendet Bianku sein Prüfschema auf echte Noven ausdrücklich nicht an: „I do not rule out the possibility that the Court might deem it necessary to conduct an ex novo analysis itself, on account of a change of circumstances, after the national authorities have reached their conclusions. " ${ }^{\circ 99}$ Sein Vorschlag lässt sich jedoch mutatis mutandis auch auf diese Konstellation übertragen.

Wie oben gesehen garantiert Art. 3 EMRK in prozeduraler Hinsicht, dass innerstaatlich Verfahrenswege bestehen, mit welchen einer maßgeblichen Veränderung des mit einer Abschiebung verbundenen Risikos Rechnung getragen werden kann. ${ }^{100}$ Im Sinne der Bianku-Logik könnte der EGMR deshalb im Hinblick auf ihm vorgelegte echte Noven zunächst untersuchen, ob innerstaatliche Verfahrenswege bestehen, mit welchen eine Beschwerdeführerin diese einer (im Hinblick auf die Anforderungen von Art. 3 EMRK effektiven) Prüfung durch die nationalen Behörden zuführen könnte. Der Umgang mit den echten Noven im Verfahren vor dem EGMR würde sich nun maßgeblich danach richten, ob der EGMR diese Frage bejahen könnte oder verneinen müsste:

Wäre die Frage zu bejahen, müsste er sich im Grundsatz auf eine Prüfung der Beschwerde aus Sicht des Zeitpunkts des letztinstanzlichen innerstaatlichen Entscheids beschränken. ${ }^{101}$ Der Beschwerdeführerin stünde jedoch offen, ein innerstaatliches Wiedererwägungsverfahren anzustoßen und nach Abschluss jenes Verfahrens eine weitere Beschwerde an den EGMR zu richten. Sofern der EGMR zu diesem Zeitpunkt noch nicht über die ursprüngliche Beschwerde entschieden hätte, würde es sich aufdrängen, die beiden Verfahren zu vereinigen. Maßgeblich wäre dann der Zeitpunkt des letzten innerstaatlichen Entscheids. Wäre die Frage hingegen zu verneinen, könnte der EGMR einerseits auf eine prozedurale Verletzung von Art. 3 EMRK schließen. Anderseits wäre er subsidiaritätsrechtlich gesehen nicht nur befugt, sondern sogar gehalten, eine vollumfängliche de-novo-Prüfung aus Sicht des Zeitpunkts seines Urteils durchzuführen, weil ein effektiver Schutz der Konventionsrechte aufgrund der Defizienzen des innerstaatlichen Verfahrensrechts nur so sichergestellt werden könnte.

Ein solches gestuftes Vorgehen erfordert vom EGMR die Untersuchung der Kompatibilität der nationalen Verfahrensordnungen mit den Vorgaben der EMRK. Dies ist jedoch keine Aufgabe, die dem EGMR fremd wäre: Bei der Prüfung der Zulässigkeitsvoraussetzungen ist er im Hinblick auf den Ausschöpfungsgrundsatz (Art. 35 Ziff. 1 EMRK) im Gegenteil sogar oft zur Prüfung der Frage berufen, ob eine Beschwerdeführerin effektive innerstaatliche Rechtsmittel zur Verfügung gehabt hätte oder aber zu Recht direkt an den EGMR gelangt ist. ${ }^{102}$ Ganz abgesehen davon ist es

\footnotetext{
${ }^{99}$ Vgl. EGMR (Große Kammer), Urteil vom 23. August 2016, J.K. v. Schweden, Concurring Opinion Bianku.

${ }^{100}$ Vgl. oben, V. B. 1.

${ }^{101}$ Auch in der Rechtsprechung des EGMR gibt es Anzeichen für einen solchen Paradigmenwechsel; vgl. (allerdings zu Art. 4 EMRK) EGMR, Urteil vom 20. Juni 2017, M.O. v. Schweiz, §§ 87-93. ${ }^{102} \mathrm{Vgl}$. Lanter, S. 63 ff.
} 
für den EGMR im Hinblick auf die Bewältigung seiner Geschäftslast unabdingbar, dass innerstaatliche Verfahrenswege bestehen, die eine Beschwerde an den EGMR entbehrlich machen; soweit die nationalen Verfahrensrechtsordnungen keine ex-nunc-Prüfungen zulassen, könnte die Feststellung einer prozeduralen Verletzung von Art. 3 EMRK die betreffenden Konventionsstaaten dazu bewegen, solche Verfahren zu schaffen. ${ }^{103}$ Dieser Anreiz besteht nicht, wenn der EGMR sich unbesehen effektiver innerstaatlicher Rechtsmittel an die Stelle der nationalen Behörden stellt und in allen Fällen ex nunc die Rechtmäßigkeit ihrer Handlungen überprüft. Die vorgeschlagene Neukonzeption im Umgang mit echten Noven könnte daher auch maßgeblich dazu beitragen, den EGMR von der Aufgabe eigenständiger Tatsachenfeststellung zu entlasten. ${ }^{104}$

\footnotetext{
${ }^{103}$ Erfolgreich war die Feststellung prozeduraler Verletzung beispielsweise im Falle Bulgariens, das lange Zeit keine effektiven Verfahrenswege im Zusammenhang mit der Rüge unmenschlicher Haftbedingungen kannte (vgl. EGMR, Urteil vom 27. Januar 2015, Neshkov und andere v. Bulgarien, §§ 177-213 und §§ 281-289); nach Feststellung einer Verletzung von Art. 3 EMRK in jenem Verfahren hat der bulgarische Gesetzgeber sein Verfahrensrecht an die Vorgaben des EGMR angepasst, was Beschwerdeführerinnen in Zukunft verpflichtet, zunächst innerstaatlich vorzugehen; vgl. EGMR, Entscheidung vom 20. Juli 2017, Atanasov v. Bulgarien und Apostolov v. Bulgarien. ${ }^{104} \mathrm{Vgl}$. in diesem Zusammenhang die allgemeinen Überlegungen von Helfer, S. 133 ff. und S. $142 \mathrm{ff}$.
}

Open Access Dieses Kapitel wird unter der Creative Commons Namensnennung 4.0 International Lizenz (http://creativecommons.org/licenses/by/4.0/deed.de) veröffentlicht, welche die Nutzung, Vervielfältigung, Bearbeitung, Verbreitung und Wiedergabe in jeglichem Medium und Format erlaubt, sofern Sie den/die ursprünglichen Autor(en) und die Quelle ordnungsgemäß nennen, einen Link zur Creative Commons Lizenz beifügen und angeben, ob Änderungen vorgenommen wurden.

Die in diesem Kapitel enthaltenen Bilder und sonstiges Drittmaterial unterliegen ebenfalls der genannten Creative Commons Lizenz, sofern sich aus der Abbildungslegende nichts anderes ergibt. Sofern das betreffende Material nicht unter der genannten Creative Commons Lizenz steht und die betreffende Handlung nicht nach gesetzlichen Vorschriften erlaubt ist, ist für die oben aufgeführten Weiterverwendungen des Materials die Einwilligung des jeweiligen Rechteinhabers einzuholen. 